\title{
Um Ambiente para o Aprendizado de Lógica de Programação Voltado a Alunos Ouvintes e não Ouvintes
}

\author{
Carla Odete Balestro Silva ${ }^{1}$, Luís Felipe dos Santos Vasconcelos ${ }^{2}$, Sandro José \\ Ribeiro da Silva ${ }^{3}$
}

1 Instituto Federal de Educação, Ciência e Tecnologia do Rio Grande do Sul (IFRS), Campus Canoas, Canoas, Rio Grande do Sul, Brasil

carla.silva@canoas.ifrs.edu.br, lipevasconcelos-11@hotmail.com, sandro.silva@canoas.ifrs.edu.br

\begin{abstract}
Programming logic studies are essential for those wishing to enter the computer field, however, about $60 \%$ of retentions in the computer course are related to this discipline. Given the retention rates cited, a study environment called TriLogic was developed. It has several playful activities, aiming at understanding the basic concepts of the subject. The project obtained 90.9\% approval from the students who used it, as well as an increase in performance in the subject. However, the gamified environment ends up not covering a portion of students, non-hearing students, who unfortunately are unable to enjoy the TriLogic environment due to their hearing condition. Given the improvements provided by the project, it was thought of an expansion in order to make the environment accessible to non-hearing students, being developed from the use of the Vlibras Suite, responsible for translating the didactic texts present in TriLogic with the help of a 3D. The system has gone through a test fase so as after validation, it could be delivered to the final user.
\end{abstract}

Resumo. Os estudos de lógica de programação são essenciais para aqueles que desejam ingressar na área de informática, contudo, cerca de $60 \%$ das retenções no curso de informática estão relacionadas a essa disciplina. Tendo em vista os indices de retenções citados, desenvolveu-se um ambiente de estudos intitulado TriLogic. $O$ mesmo conta com diversas atividades lúdicas, visando à compreensão de conceitos básicos da disciplina. O projeto obteve uma aprovação de 90,9\% dos alunos que o utilizaram, bem como um aumento do rendimento na disciplina. No entanto, o ambiente gamificado acabava não abrangendo uma parcela dos estudantes, alunos não ouvintes, os quais, infelizmente, não eram capazes de usufruir do ambiente TriLogic devido a sua condição auditiva. Diante das melhorias proporcionadas pelo projeto, pensouse em uma ampliação com o intuito de tornar o ambiente acessível a alunos não ouvintes, sendo desenvolvida a partir da utilização da Suite Vlibras, responsável por realizar a tradução dos textos didáticos presentes no TriLogic com o auxílio de um intérprete 3D. O sistema passou por uma etapa de teste para que após a validação, o mesmo pudesse ser entregue ao usuário final.

\section{Introdução}

Para realizar o ingresso na área da informática, é essencial que o futuro profissional já tenha iniciado sua jornada nos estudos de informática básica e principalmente lógica de 
programação. Tendo em vista que, "Lógica de programação é requisito fundamental nos cursos de computação", segundo Rocha (2010), dela depende o entendimento de qualquer linguagem de programação, em que a compreensão da disciplina tem reflexos no desempenho do aluno na aprendizagem de novos conteúdos.

No entanto, como é apresentado também por Rocha (2010), quase 60\% das retenções no curso de informática estão relacionadas à disciplina de lógica de programação, resultados segundo o autor, que podem estar relacionados à falta de motivação dos alunos frente a uma dificuldade, ou até mesmo ao ritmo do professor ser diferente do ritmo de assimilação do aluno.

A lógica de programação é uma disciplina ofertada no primeiro ano dos cursos técnico em nível médio e no primeiro semestre dos cursos superiores de desenvolvimento de sistemas do Instituto Federal de Educação, Ciência e Tecnologia do Rio Grande do Sul Campus Canoas. E assim, como os estudos de Rocha (2010) apontam, o componente curricular é a responsável pela maior parte das retenções do curso.

Existem diversas estratégias possíveis para a reversão desses altos índices de retenções apresentados, uma delas é denominada gamificação, definida por Kapp(2014) como, "o uso de mecânicas, estéticas e pensamentos dos games para engajar pessoas, motivar a ação, promover a aprendizagem e resolver problemas", assim, mecânicas de jogos seriam motivadores para a resolução de problemas de qualquer área do conhecimento, inclusive a lógica de programação.

Tendo conhecimento dos benefícios da utilização da gamificação, o projeto de pesquisa Uso de Técnicas de Gamificação como Auxílio ao Ensino e Aprendizagem de Lógica de Programação desenvolveu um ambiente a partir das técnicas de gamificação, denominado TriLogic.

Iniciado em 2016 no Campus Canoas, o ambiente

[...] conta com exercícios lúdicos no intuito de estimular o aluno a compreender conceitos básicos em um contexto geral, sobre descrição narrativa, fluxogramas, manipulação de variáveis, utilização de tabelas com a introdução dos conceitos de vetores e matrizes, cláusulas condicionais (se/então/senão) e estruturas de repetição (para/repita-até e faça-enquanto)

(BARBOSA; MUCH; SILVA, C.; BIGOLIN; SILVA, S.; 2017).

Com o intuito de fazer os alunos terem um contato mais amigável com a lógica de programação, o ambiente gamificado proposto pelo projeto de pesquisa foi essencial para ajudar a evitar os problemas mais apontados por Rocha (2010), assim, o aluno consegue aplicar os conhecimentos obtidos em cada fase do ambiente, o estimulando a continuar aprendendo. Dessa forma, o mesmo se mostrou muito importante no processo de aprendizado dos alunos, como apontado pelo coordenador do projeto MSc Sandro Silva (2016) alunos participantes do projeto obtiveram "[...] melhora acima de $20 \%$ em seu desempenho na disciplina de Lógica de Programação".

Embora os resultados do uso do TriLogic tenham sido promissores, o ambiente inicialmente não abrangia uma parcela dos estudantes, alunos não ouvintes, infelizmente, não eram capazes de usufruir plenamente do ambiente TriLogic devido a sua condição auditiva. Os não ouvintes tem como primeiro idioma a Língua Brasileira de Sinais, doravante Libras e o Português Escrito como idioma secundário, conhecida como L2. 
Logo, as atividades propostas pelo ambiente se tornavam inacessíveis a eles já que estão organizadas com ênfase na produção textual. Segundo Stumpf (2000) explica que "a produção escrita dos surdos é quase inexistente [...] Na leitura, mesmo após muitos anos de escolaridade, a compreensão é pequena".

\section{Educação Inclusiva para Não Ouvintes}

Para os não ouvintes, a realidade na qual os mesmos estavam inseridos começou a adquirir traços mais inclusivos a partir do ano de 1755. Neste período, o francês Charles Michel de I'Épée introduz o primeiro método de ensino voltado a pessoas não ouvintes $(\mathrm{CHIH}$, 2013). Por volta de 1857 o Brasil realizou seu primeiro contato com uma educação voltada para o público deficiente. Como apresentado por Pereira (2019) no ano de 1855, o professor não ouvinte Hernest Huet natural da França, chega a cidade do Rio de Janeiro e após dois anos no país, funda o Instituto Imperial para Surdos-Mudos, primeira instituição voltada ao ensino de deficientes auditivos no Brasil. Passados alguns anos, a instituição sofreu uma renomeação, ficando conhecida como Instituto Nacional de Educação para Surdos (INES). Além disso, o INES se tornou responsável por desenvolver, disseminar e estabelecer a LIBRAS no país (LIBRAS, 2018).

Por conseguinte, diversos direitos voltados a pessoas não ouvintes foram estabelecidos no Brasil, como o Decreto $n^{\circ} 5626$, responsável por garantir uma educação bilíngue em salas regulares com a presença de intérpretes, este documento afirma que "A Libras deve ser inserida como disciplina curricular obrigatória nos cursos de formação de professores para o exercício do magistério, em nível médio e superior” (BRASIL, 2005).

\section{Tecnologias e Ferramentas adotadas}

Para o desenvolvimento da adaptação do ambiente TriLogic optou-se pela utilização da linguagem interpretada Java Script (JS) como linguagem de programação. O JS tem como objetivo tornar determinados processos do desenvolvimento WEB mais dinâmicos, auxiliando na adaptação das páginas e trazendo funcionalidades mais complexas à mesma (SILVA, G. 2015). Também foram necessárias adaptações nos arquivos JavaScript Object Notation (JSON) presentes no TriLogic, para que assim fosse possível realizar a tradução do ambiente.

Em conjunto, a linguagem de marcação Hypertext Markup Language (HTML) mostrou-se eficaz para a unificação dos códigos referentes à janela de exibição do Vlibras ao código proveniente das telas do ambiente gamificado, segundo Eis (2011) "O HTML é a linguagem base da internet [...] utilizada para desenvolver websites". Ademais, a linguagem de estilização de páginas Cascading Style Sheets (CSS) foi efetiva na padronização do tamanho dos objetos que estariam visíveis aos alunos no ambiente, bem como para destacar os textos a serem traduzidos.

Para que o ambiente TriLogic se tornasse acessível a alunos não ouvintes, foram estudadas diversas ferramentas voltadas à tradução online do português escrito para Libras. Os seguintes aspectos foram levados em consideração para a seleção das ferramentas de tradução: o número de usuários da ferramenta, estabilidade do projeto no mercado, bem como, o fato de possuírem um intérprete 3D para a comunicação com o usuário.

Considerando os aspectos pontuados durante o desenvolvimento da análise de diversas ferramentas, nota-se que dentre as avaliadas, a que demonstrou maior potencial 
para suprir as necessidades do projeto denomina-se suíte Vlibras. Um dos fatores que mais pontuou a seu favor, está relacionado ao fato do mesmo possuir código livre, assim, as ferramentas necessárias puderam ser implementadas ao ambiente de maneira gratuita sem perda de qualidade. Sistemas como a suíte Vlibras, se tornam essenciais para inclusão de pessoas não ouvintes dentro da atual sociedade. Logo, através das ferramentas disponibilizadas pelo suíte, sua integração ao TriLogic foi responsável por acrescer ainda mais ao ambiente, tornando possível o aumento da diversidade do público atingido pelo projeto.

\section{TriLogic junto a acessibilidade}

A partir da ampliação realizada no ambiente gamificado TriLogic, o mesmo se tornou acessível tanto aos alunos ouvintes, quanto aos alunos não ouvintes. Sendo assim, após o login no sistema, o aluno do TriLogic poderá visualizar a tela que representa o menu inicial do ambiente e, a partir desse ponto, conseguirá iniciar suas atividades no ambiente gamificado presente na Figura 1.

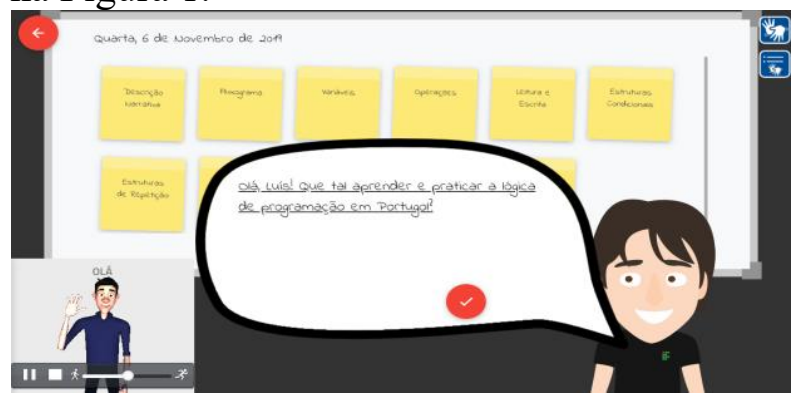

Figura 1. Tela inicial TriLogic pós adaptação

A Figura 1 apresentada acima é composta por uma série de elementos responsáveis por representar as funcionalidades do ambiente TriLogic. Começando pelo agente animado encontrado na região inferior esquerdo, para Reategui (2006), um agente é responsável por monitorar a interação do usuário com o sistema, utilizando dados coletados para realizar recomendações personalizadas, sendo assim, no TriLogic utilizase uma caixa de diálogo para a interação entre o ambiente e o aluno. Em seguido pode-se visualizar ao fundo diversos post-its com os tópicos presentes no ambiente dispostos em um quadro branco, cada um deles representa um exercício diferente.

No entanto, as funcionalidades acrescidas ao ambiente TriLogic responsáveis por auxiliar na adaptação do mesmo aos usuários não ouvintes, estão presentes na região superior da lateral direita da Figura 1. Começando pelo ícone de acessibilidade presente na Figura 2.

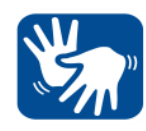

Figura 2. Ícone de acessibilidade em Libras

Ao clicar no ícone de acessibilidade, o intérprete 3D Ícaro é carregado na região inferior esquerda do ambiente, como apresentado na Figura 1. Quando o intérprete está ativo, à medida em que o usuário percorre o ambiente com o mouse os trechos de textos se tornam sublinhados. O usuário deve parar o cursor sobre o texto desejado para dar início à tradução do português escrito para Libras. Os sinais em Libras serão apresentados ao aluno através do intérprete Ícaro. 
Através da janela de tradução, além de poder visualizar os sinais, o aluno poderá usufruir das funcionalidades disponíveis na barra de ferramentas presente na região inferior da área de tradução. A partir dela, faz-se possível pausar ou prosseguir com a tradução, finalizar a tradução e aumentar ou diminuir a velocidade dos sinais apresentados. Tais funcionalidades foram descritas da esquerda para a direita, na ordem em que são representadas pela Figura 1, Ao final de cada tradução, a mesma pode ser realizada novamente.

Junto ao ícone de acessibilidade faz-se presente no TriLogic uma funcionalidade que auxiliará na tradução do ambiente gamificado, tal funcionalidade é representada pelo ícone apresentado na Figura 3.

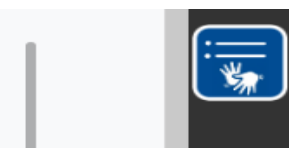

Figura 3. Ícone de Funcionalidade

O desenvolvimento dessa funcionalidade, ocorreu através de uma parceria com a intérprete de libras presente no Campus Canoas. Por meio desta parceria, a intérprete testou a aplicação e sugeriu algumas melhorias. Em decorrência dos apontamentos realizados pela mesma, notou-se que o intérprete Vlibras não possuía uma série de sinais para palavras relacionadas à área da informática. A partir da ausência de um sinal específico para uma palavra, o intérprete realiza a datilologia da mesma, ou seja, a datilologia equivale à soletração de uma palavra.

Contudo, segundo a profissional de Libras, em algumas situações a datilologia pode confundir os alunos, devido ao fato das palavras presentes na área técnica não serem tão recorrentes no cotidiano dos mesmos. Tendo em vista a solução desse problema, desenvolveu-se um glossário de sinais voltados para informática. Sendo assim, ao passar o cursor do mouse sobre o menu de sinais, um submenu denominado glossário é apresentado na tela. Desta forma, quando o aluno desejar visualizar as palavras presentes no glossário, basta acessá-lo através de um clique no submenu. Tal ação faz com que uma modal de conteúdo seja apresentada na tela principal, a qual pode ser visualizada na Figura 4.

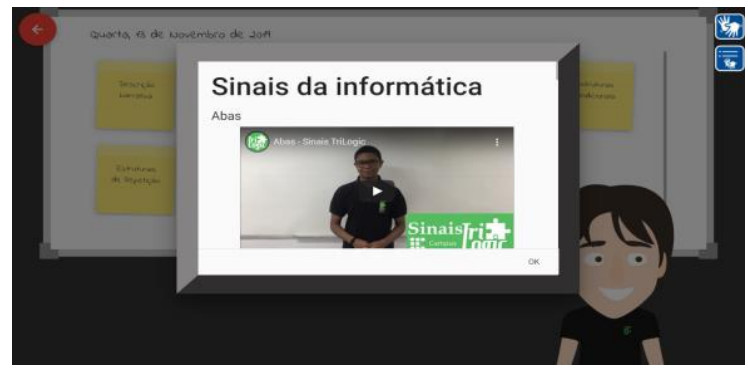

Figura 4. Modal do glossário

Através da Figura 4, percebe-se que a modal responsável por armazenar os sinais presentes no glossário é composta por um título, denominado "Sinais da informática" e uma legenda com a palavra a ser representada no vídeo logo abaixo.

Antes de serem incorporados ao glossário do ambiente gamificado, todos os vídeos gravados foram analisados e aprovados pela intérprete de libras. Contudo, é de grande importância ressaltar a origem dos vídeos utilizados como referência para a produção do material presente no glossário do ambiente TriLogic. Os vídeos 
compartilhados são provenientes de um projeto realizado no Instituto Federal de Educação, Ciência e Tecnologia da Paraíba (IFPB) Campus Campina Grande. Para o desenvolvimento dos vídeos presentes no ambiente foram utilizados os sinais provenientes da região nordeste do país, pois não foram criados sinais específicos da informática no estado do RS.

\section{Conclusão}

Para a efetivação da validação do ambiente TriLogic, realizou-se uma entrevista semiestruturada com a profissional de Libras atuante no IFRS Campus Canoas. Por meio da entrevista, a intérprete pôde ofertar uma série de informações necessárias sobre a usabilidade do ambiente gamificado e sobre a relevância do presente trabalho para a vida acadêmica dos alunos não ouvintes.

Um dos fatores responsáveis por contribuir para a escolha deste tipo de validação, está relacionado ao baixo número de usuários que se encaixam ao público alvo do ambiente. Com o intuito de embasar tal afirmação, dados disponibilizados pelo registro de estudantes do IFRS Campus Canoas elucidaram a situação referente ao ingresso e evasão de alunos não ouvintes. Através desses, notou-se que no segundo semestre de 2019, período no qual o projeto estava em desenvolvimento, apenas dois alunos não ouvintes estavam vinculados a instituição dentro do curso de Análise e Desenvolvimento de Sistemas. Contudo ambos os estudantes não compareciam as aulas, impossibilitando suas participações na validação das ampliações do ambiente TriLogic.

Por intermédio dessa situação, a entrevista realizada com a interprete foi confeccionada a partir do desenvolvimento de sete perguntas, dentre as quais uma consiste no seguinte questionamento "Você gostaria de ressaltar alguma funcionalidade específica presente no ambiente?".

A partir da questão apresentada, a interprete ressaltou o fato do ambiente gamificado possuir uma interatividade agradável entre o usuário e o sistema. $\mathrm{O}$ mesmo se reflete na utilização por alunos não ouvintes "sobre a adaptação, eu gostei do fato de poder fazer uma tradução várias vezes, através da barra de funcionalidades do Vlibras." (ENTREVISTADA, 2019). Dessa forma, pode-se concluir que a plena interatividade entre o ambiente e ambos os usuários se faz importante para a utilização do sistema.

Diante dos dados provenientes da entrevista, constatou-se a aprovação do trabalho desenvolvido a partir de sua proposta inicial, de acordo com uma opinião profissional da área de Libras. Diversos aspectos debatidos inicialmente com a intérprete serviram como embasamento para melhorias no ambiente, e após a realização da entrevista, mostraramse válidos. Portanto, por meio da aprovação da intérprete, o projeto de pesquisa TriLogic tornou-se com sucesso apto a atender alunos ouvintes e não ouvintes.

Dessa maneira, a inexistência de alunos não ouvintes na instituição no momento dos testes não desmerece a necessidade de adaptação de um sistema para o ensino de quaisquer conteúdos para os alunos com essa deficiência. Como observado, dois alunos não ouvintes já se matricularam no curso superior em Análise e Desenvolvimento de Sistemas, mas não continuaram os estudos por motivos desconhecidos. Antecipar-se às necessidades dos alunos portadores de deficiência tornando os ambientes acessíveis, é um passo importante na verdadeira inclusão pretendida nos Institutos Federais e demais instituições de ensino. Assim percebe-se que a inclusão não deve ser vista como algo extracurricular, mas sim, como uma atividade necessária em todos os segmentos. 


\section{Referências}

Barbosa, Brenda Anghinoni; Much, Bruno; Silva, Carla; Bigolin, Marcio; Silva, Sandro. (2017)" Tri-Logic - Proposta Lúdica Gamificada para o Ensino e Aprendizagem da Lógica de Programação com o Uso da Mineração de Dados como Ferramenta de Auxílio ao Professor. Canoas, Rs”. Congresso brasileiro de informática na educação, 6., 2017, Recife.: Sbie, 2017. 1754 p.

Brasil. (2005) "Decreto $\mathrm{n}^{\mathrm{o}} 5.626$, de 22 de dezembro de 2005". Regulamenta a lei $\mathrm{n}^{\mathrm{o}}$ 10.436, de 24 de abril de 2002, que dispõe sobre a Língua Brasileira de Sinais - Libras, e o art.

Silva, Giancarlo. (2015) “O que é e como funciona a linguagem JavaScript?”. Canaltech, Disponível em: https://canaltech.com.br/internet/O-que-e-e-comofunciona-alinguagem-JavaScript/.

Chih, Chung Ting. (2013) "Um pouco da história da língua de sinais no mundo e no Brasil.”. Diversidade em Comunicar. Disponível em: https://diversidadeemcomunicar.wordpress.com

Eis , Diego.(2011) “O básico: O que é HTML?: Entenda o HTML básico, saiba o que significa tags do HTML e entenda como fazer." . [S. 1.]. Disponível em: https://tableless.com.br/o-que-html-basico/.

Kapp. K. M. Blair, L. and Mesch, R. (2014) "The Gamification od Learning and Instruction - Fieldbook. Ideas into Practice". San Francisco. Willey.

Libras. (2018) "INES”. [S. 1.]: LIBRAS. Disponível em: http://www.libras.com.br/ines.

Pereira, Andresa Vaniele Barbosa. (2019) "Língua brasileira de sinais: História da Libras, porque estudar, como atender um aluno surdo”. [S. 1.]: Brasil Escola. Disponível em: https://meuartigo.brasilescola.uol.com.br/educacao/lingua-brasileirasinais.htm.

Reategui, Eliseo Berni et al. (2006) "um agente animado para ambientes de aprendizagem colaborativos". Revista Brasileira de Informática na Educação, 2 Instituto de Informática - UFRGS, v. 14, ed. 3, p. 28-38, 2006. Disponível em: https://www.brie.org/pub/index.php/rbie/article/view/27/22.

Rocha, P. S. et al. (2010) "Ensino e Aprendizagem de Programação: Análise da Aplicação de Proposta Metodológica Baseada no Sistema Personalizado de Ensino”. RENOTE Revista Novas Tecnologias na Educação, v. 8, n. 3, p. 1-11.

Silva, Sandro José Ribeiro. (2016) "O Ensino e Aprendizagem da Lógica de Programação através da Linguagem LUA em uma proposta Gamificada com auxílio da Mineração de Dados Educacionais como suporte ao professor”. Dissertação - Unisinos, Brasil,

Stumpf, Marianne Rossi. (2000) "Língua de sinais: escrita dos surdos na Internet. PorSinal". Disponível em: http://www.porsinal.pt/index.php?ps=artigos\&idt=artc\&cat=15\&idart=109 